\title{
On Gold Catalysis and Beyond...
}

\author{
Estibaliz Merino and Cristina Nevado $\S^{\star}$
}

\$Werner Prize Winner 2013

\begin{abstract}
Over the past six years, our research group has explored the combination of new transformations based on gold catalysis with their application towards the synthesis of complex organic molecules including natural products. This work was rewarded with the 2013 Werner Prize from the Swiss Chemical Society.
\end{abstract}

Keywords: Gold catalysis · Reaction mechanism · Total synthesis

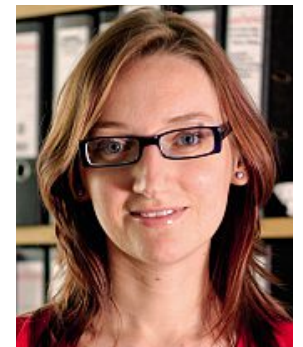

Cristina Nevado was born in 1977 in Madrid, where she graduated in chemistry at the Autónoma University in 2000 . In October 2004 she received her $\mathrm{PhD}$ in organic chemistry at the same university working with Prof. Antonio M. Echavarren in the cyclization of enynes catalyzed by platinum and gold complexes. In December 2006 she joined the group of Prof. Alois Fürstner at the Max-Planck-Institut für Kohlenforschung (Germany) where she was part of the team who conquered the first total synthesis of Iejimalide B, a marine macrolide possessing a very sensitive architecture. In May 2007 she started her independent career as an Assistant Professor at the University of Zürich. In 2011, Cristina was awarded the Chemical Society Reviews Emerging Investigator Award and the Thieme Chemistry Journal Award in recognition of her contributions in the field of synthetic organic chemistry. In 2012 she received an ERC Junior Investigator grant and was awarded the Werner Prize of the Swiss Chemical Society in 2013. This year she become Full Professor at the Organic Chemistry Institute of the University of Zürich. Rooted in the wide area of organic chemistry, her research program is focused on complex chemical synthesis and new organometallic reactions.

\section{Introduction}

Synthetic chemistry is a fascinating discipline grounded on the synergistic combination of different areas of expertise. The ability to design and synthesize complex molecular structures with exquisite control, not only over the atomic composition and the assembly process, but also over additional parameters such as molecular shape, aggregation and function, are just some of the many abilities expected from a synthetic chemist. One might have to admit though, that despite the enormous potential held by this already mature field, some of the processes currently used in laboratory settings for the construction of medium to complex chemical blueprints still remain inefficient. Thus, novel methods to accomplish the always-evolving structural challenges posed by closely related disciplines such as biology, medicine or pharmacy are needed. Our research revolves around the discovery and development of new reactions mediated by late transition metals, particularly gold. The goal is not only to provide new synthetic tools to the community but also to understand the mechanistic features governing these processes so that alternative, even more challenging transformations, can be tackled. A major validation of this approach stems from the application of these new methodologies towards the synthesis of complex organic molecules. In this paper, we summarize some of these research lines including, among others, ${ }^{[1]}$ the development of new gold-catalyzed reactions and the synthesis of natural products. ${ }^{[2]}$

\section{On Gold as a Catalytic Lewis Acid}

The Lewis acid character of gold(I) and gold(III) complexes was identified several decades ago. The strong relativistic effects governing gold's coordination chemistry, and its thus favorable interaction with $\pi$-unsaturated systems, contribute to the so called 'carbophilicity' defining its catalytic activity. ${ }^{[3]}$ The synthetic potential behind the activation of carbon unsaturated moieties (alkenes, alkynes, allenes, etc...) has been extensively exploited in the past years. ${ }^{[4]}$ A particularly productive field is the activation of propargyl carboxylates (I) towards 1,2-migration of the acyl group and/or Claisen [3,3]-sigmatropic rearrangements (Scheme 1).[5] Terminal alkynes or alkynes substituted with electron withdrawing groups lead to carbene type intermediates (III) via 1,2-migration of the acyl group. In contrast, internal alkynes lead to allenyl acetates $(\mathbf{V})$ by migration of

\footnotetext{
${ }^{*}$ Correspondence: Prof. Dr. C. Nevado

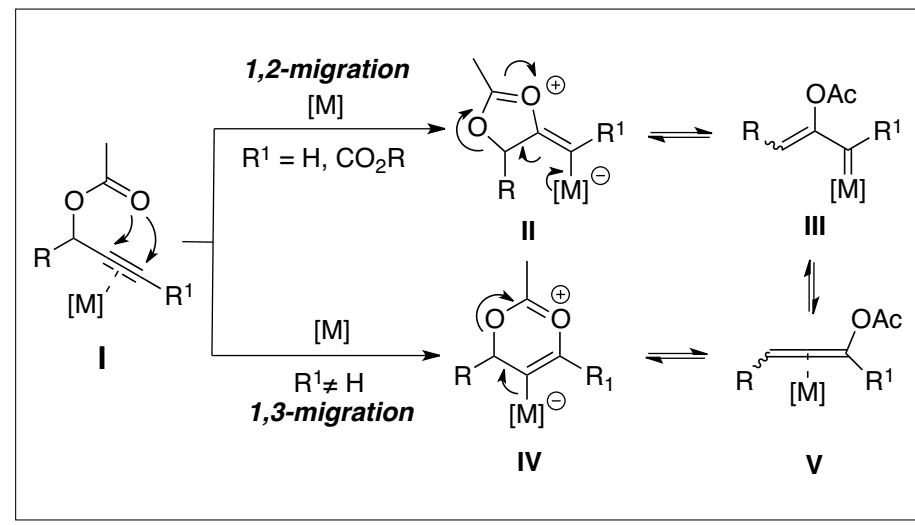

Scheme 1.

Reactivity of propargyl carboxylates in the presence of late transition metals. 
the carboxylate group to the terminal carbon atom of the triple bond. These two reaction pathways are not independent, and a second migration of the acyl group in III could also produce intermediates of type V (Scheme 1). The mechanistic scenario summarized in Scheme 1 represents a generalization and several exceptions to these common reactivity patterns have been described, some of them stemming from our own program.

\section{A Stereoselective Synthesis of 2,3-Bis-acetoxy-1,3-dienes}

We envisioned that the intramolecular 1,2-migration of the carboxylate group in III could be further exploited. Thus, propargylic 1,4-bisacetates (1) were synthesized and their reactivity tested in the presence of catalytic amounts of gold complexes producing 2,3-bisacetoxy-1,3dienes in a highly stereocontrolled manner (Scheme 2).[6] The reaction is proposed to proceed via gold-carbene 2 formed upon initial 1,2-migration of one of the acetoxy groups in the starting material. A second migration of the same group in $\mathbf{2}$ to give the 1,3-allenyl acetate is disfavored due to the presence of a second ester group at the $\alpha$-position of the carbenic carbon. Migration of this second acyl moiety affords bis-acetoxy dienes $\mathbf{3}$ and $\mathbf{4}$ (Scheme 2).

In contrast to the general behavior expected for internal alkynes and shown in the bottom half of Scheme 1 (i.e. 1,3-migration path), carbene type intermediates 2 seem to account for the formation of the observed products in these transformations, thus showcasing the stepwise nature of propargyl acetate migrations. The ancillary ligand on gold plays a key role in these transformations: (1Z,3Z)-dienes (3) are selectively obtained with IPrAuNTf whereas (1Z,3E)-1,3-dienes (4) are produced with $\mathrm{Ph}_{3}$ PAuNTf ${ }_{2}$ as catalyst.

\section{Gold-catalyzed Cyclopenta- and Cyclohexannulation Reactions}

The versatility of the intermediates generated during the gold-catalyzed rearrangement of propargyl acetates prompted us to explore new substrates incorporating a cyclopropyl substituent

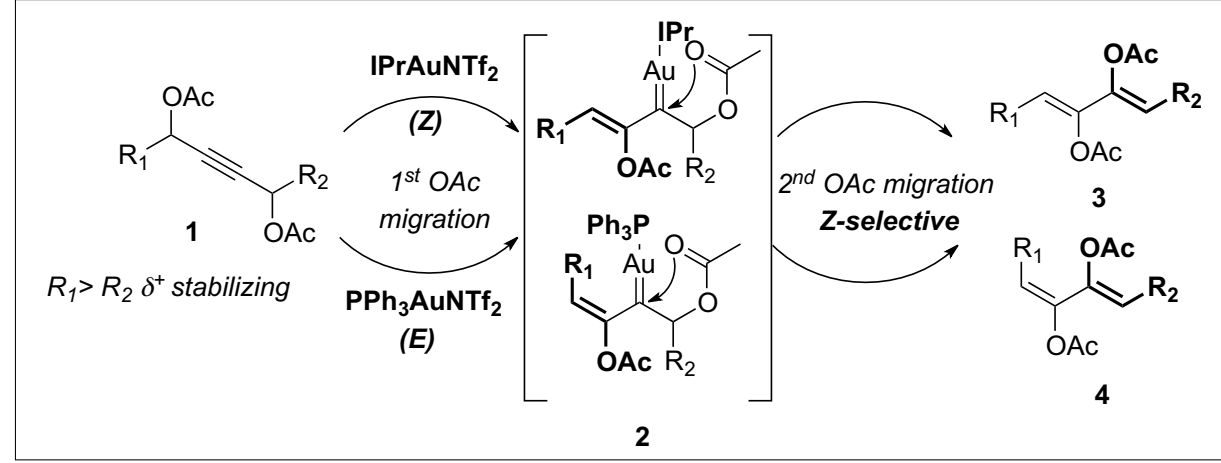

Scheme 2. Stereocontrolled synthesis of 2,3-bis-acetoxy-1,3-dienes.
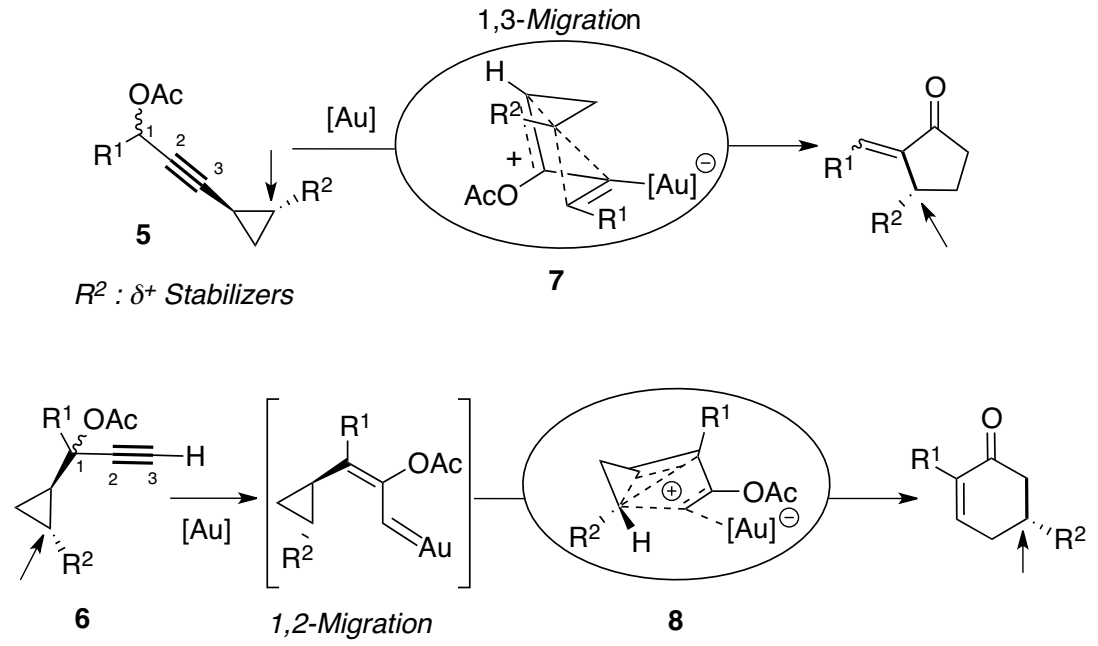

Gold-stabilized carbocations = Stereospecific transformations

Scheme 3. Cycloisomerizations of 3- and 1-cyclopropylpropargyl acetates. in a collaboration project with Givaudan AG. As shown in Scheme 3, compounds 5 and 6 were selected as benchmark substrates. ${ }^{[7]}$ Upon coordination of gold to the alkyne and 1,3- or 1,2-migration of the acetate moiety, the cyclopropyl ring undergoes a ring-opening process. A 1,5or a 1,6-gold dipole is generated ${ }^{[8]}$ which upon cyclization delivers five- and sixmembered ring terpenoids, respectively. These dipole intermediates can be better defined as 'gold-stabilized non-classical carbocations' in which gold is able to stabilize the positive electron density generated upon opening of the three membered rings as shown in intermediates 7 and 8. Although stereospecific in nature, the stereochemical information transfer from $\mathrm{C}(5)$ in the substrate onto the final products is not complete. In fact, experimental evidence and DFT calculations show that a cyclopropyl opening/closure can compete with the cyclization event. Surprisingly, such cyclopropyl scrambling has been found for both cis and trans settings, which showcases the configurational promiscuity of three-membered rings.

\section{Gold-catalyzed Cyclopenta-and Cycloheptannulation Cascade Reactions: Application Towards the Enantioselective Synthesis of Frondosin A}

We hypothesized that the so-called 'non-classical gold-stabilized carbocations' (7 and 8, Scheme 3) could constitute an efficient platform for the stereocontrolled synthesis of larger ring sizes. The group of Prof. Toste pioneered the use of in situ generated gold carbenes towards alkene cyclopropanation. ${ }^{[9]}$ Such carbene intermediates could be easily generated upon reaction of propargyl acetates $(9)$ in the presence of a catalytic amount of gold (Scheme 4). A reaction sequence was designed in which, upon 1,2-migration of the acetyl group, a cyclopropanation on a 1,3-diene (12) would take place. The metal center could then react in situ activating the vinyl acetate moiety in intermediate $\mathbf{1 1}$ inducing a homo-Cope rearrangement to produce seven-membered rings (13) in a single synthetic operation (Scheme 4, top, right pathway). Substituted cyclopentenylacetates (14) could alternatively be obtained when the diene is replaced by a simple alkene (10) under similar reaction conditions (Scheme 4, top left pathway). $[10]$

Frondosins are intriguing sesquiterpenes of marine origin, which contain a seven-membered ring in their structure. We envisioned an application of our methodology towards an enantioselective synthesis of Frondosin A by condensation of pivalate 


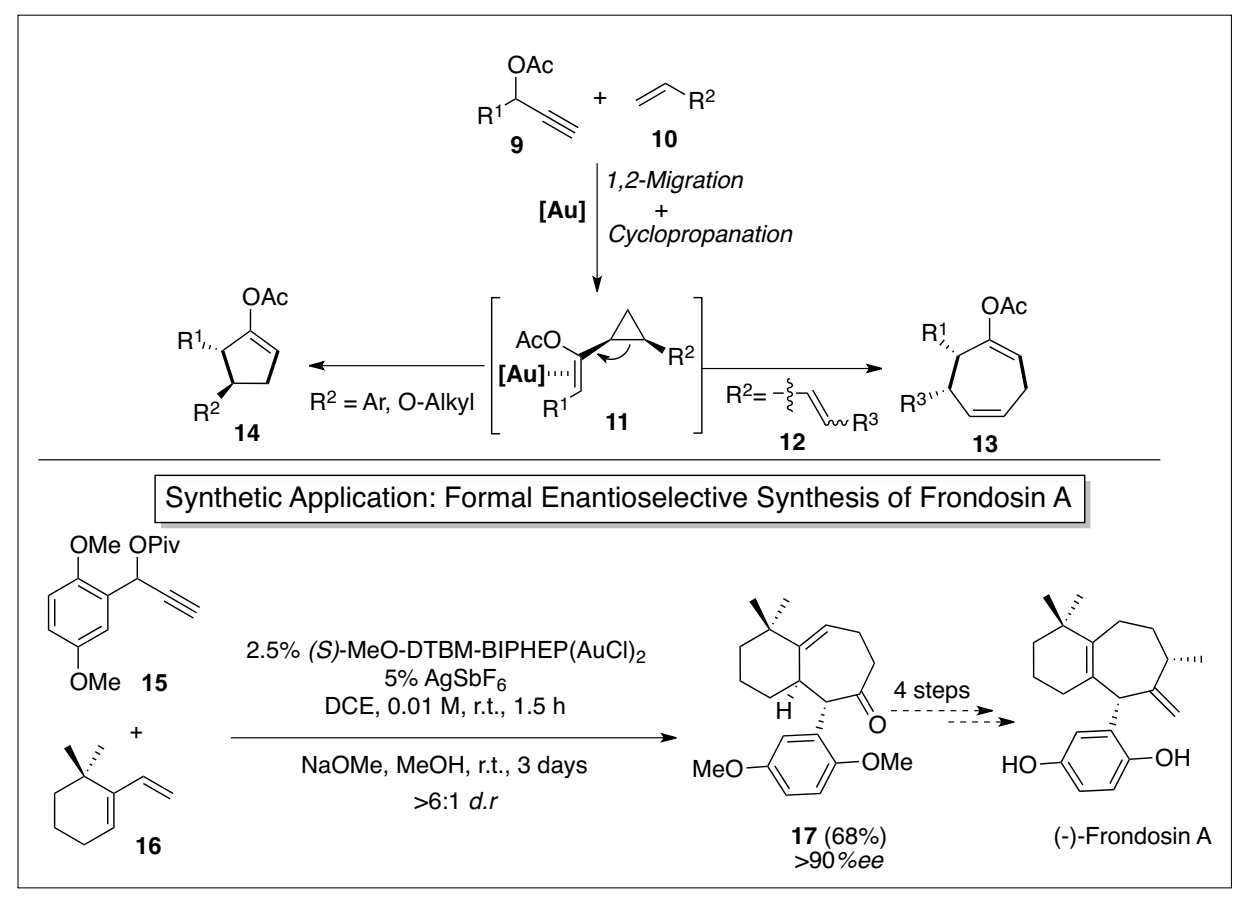

Scheme 4. Design of 1,2-acyl/cyclopropanation/cycloisomerization cascade and formal enantioselective synthesis of Frondosin A.

15 with 6,6-dimethyl-1-vinyl cyclohexene (16) (bottom part of Scheme 4). The presence of a chiral gold complex as catalyst yielded, after hydrolysis and equilibration at the $\alpha$-position of the formed ketone, compound 17. In only four steps, compound $\mathbf{1 7}$ can be elaborated into Frondosin A, so that our approach constitutes a formal enantioselective straightforward synthesis of this bicyclic natural product.[10]

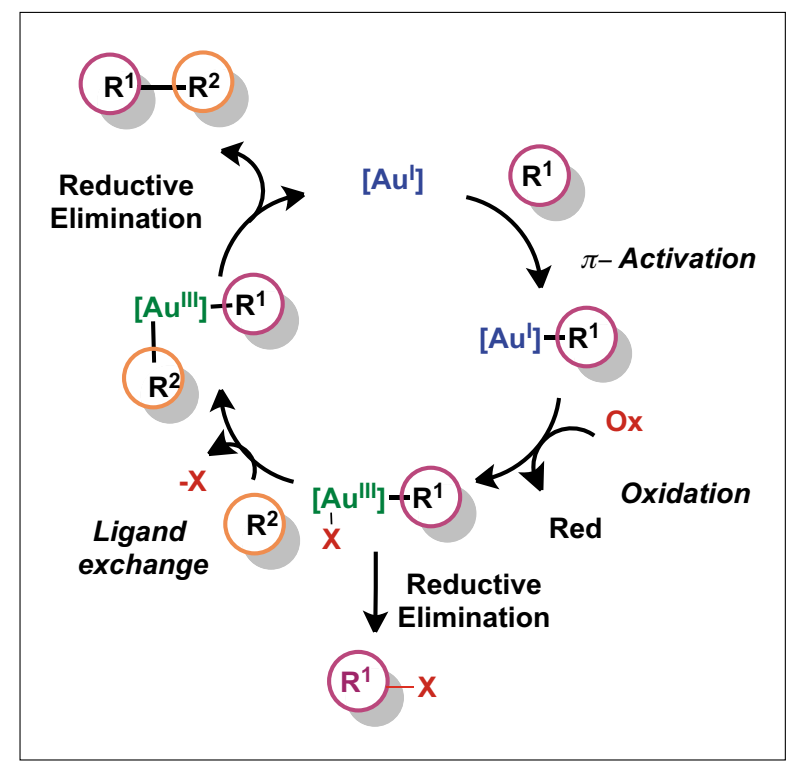

Scheme 5. Mechanistic proposal towards goldcatalyzed redox cross-coupling reactions.

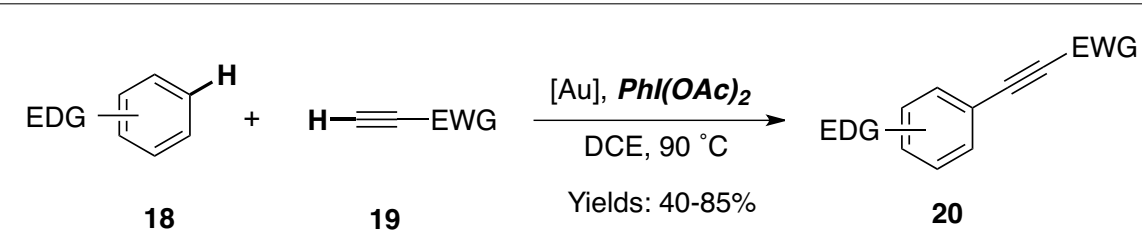

Scheme 6. Gold-catalyzed cross-coupling of arenes and terminal alkynes.
Miyaura and Sonogashira cross-coupling reactions have been described using gold salts as catalysts, ${ }^{[12]}$ the exact reaction mechanism of these transformations is still a matter of debate. ${ }^{[13]}$ However, $\mathrm{Au}(\mathrm{I}) / \mathrm{Au}(\mathrm{III})$ redox catalytic cycles have bloomed in the recent years and synthetic applications have also been devised. ${ }^{[14]}$ The most common strategy to obtain $\mathrm{Au}(\mathrm{I}) / \mathrm{Au}(\mathrm{III})$ turnovers relies on the use of a strong oxidant to enable the oxidation of the metal followed or preceded by the coordination of an additional organic fragment. Reductive elimination on a highly oxidized intermediate would be responsible for the formation of the new $\mathrm{C}-\mathrm{C}$ or C-heteroatom bond (Scheme 5). [13]

\section{Gold-catalyzed Ethynylation of Arenes}

We decided to explore this concept tackling the formation of $\mathrm{C} s p-\mathrm{C} s p^{2}$ bonds. We aimed to find an alternative method to the well-established Sonogashira crosscoupling reaction, as electron-rich aromatic rings as well as electron-deficient alkynes are usually problematic under $\mathrm{Pd}$ catalysis affording the corresponding aryl-alkyne products in low yields. We wanted to overcome these limitations with the design of a new method that would enable the coupling electron-rich aromatic substrates (18) with electrondeficient alkynes (19) through the direct functionalization of $\mathrm{C} s p^{2}-\mathrm{H}$ and $\mathrm{C} s p-\mathrm{H}$ bonds. We hypothesized that gold(I)alkynyl species would be formed in situ, in analogy to the copper acetylides found in the Sonogashira reaction. In the presence of a strong oxidant such as iodobenzene diacetate, a gold(III) intermediate would be generated. This highly electrophilic complex could then react in the presence of an electron-rich arene to give an aryl$\mathrm{Au}(\mathrm{III})$-alkynyl intermediate, which upon reductive elimination would generate the new Csp-Csp $p^{2}$ bond (20) and gold(I) to start a new catalytic cycle. The successful realization of this concept has been summarized in Scheme 6. [15]

\section{Gold-catalyzed C-F Bond Formation}

The introduction of $\mathrm{C}-\mathrm{F}$ bonds in organic molecules has found widespread use in medicinal chemistry due to the improved properties (permeability, metabolic stability, etc...) that fluorine-containing compounds display compared to their analogous $\mathrm{C}-\mathrm{H}$ counterparts. ${ }^{[16]}$ In the past years, strategies for the formation of $\mathrm{C} s p^{2}-$ and $\mathrm{C} s p^{3}-\mathrm{F}$ bonds based on transition metal-catalyzed reactions 


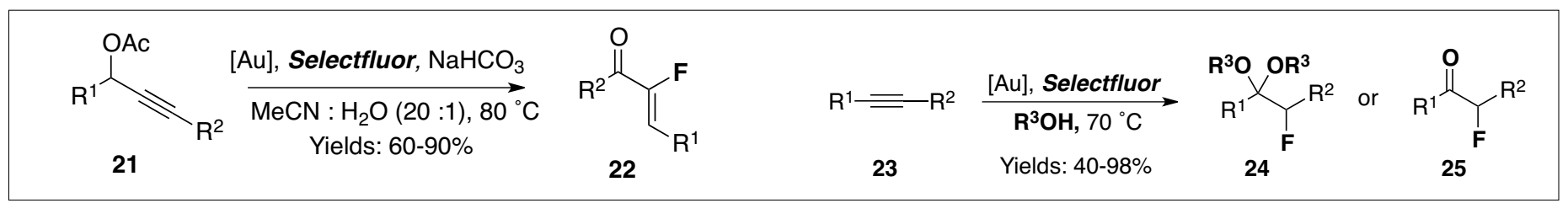

Scheme 7. Synthesis of $\alpha$-fluorenones and $\alpha$-fluoroketones and/or acetals.

have gained momentum due to the mild reaction conditions and high functional group compatibility they offer compared to analogous non-catalyzed processes. ${ }^{[17]}$

We have devised two processes for the efficient formation of $\mathrm{C}-\mathrm{F}$ bond using gold catalysts. In the first one, we used propargyl acetates as benchmark substrates, and examined their reactivity in the presence of different fluorinating reagents. Thus, the reaction of $\mathbf{2 1}$, in the presence of catalytic amounts of IPrAuNTf 2 and Selectfluor enabled the synthesis of $\alpha$-fluoroenones 22 (Scheme 7, left). ${ }^{[18]}$ A second transformation was designed with alkynes (23) as starting materials. In the presence of gold and Selectfluor, but with an alcohol as solvent, $\alpha$-fluoro acetals $\mathbf{2 4}$ and/or $\alpha$-fluoro

ketones $\mathbf{2 5}$ could be efficiently obtained in a single synthetic operation (Scheme 7 , right). ${ }^{[19]}$ The reaction is highly regioselective and amenable to both internal and terminal alkynes. Although coordination of gold to the triple bond seems to be the initial step in both processes described above, an in depth mechanistic understanding of the exact species yielding the formation of the new $\mathrm{C}-\mathrm{F}$ bonds is still lacking. Further studies along these lines are currently underway in our group.

\section{Gold-catalyzed Difunctionalization of Alkenes}

Although alkynes and alkenes can coordinate gold equally well, the number of

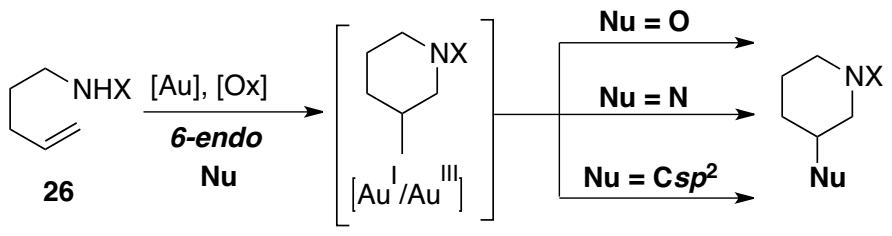

$\mathrm{Nu}=\mathrm{OH}, \mathrm{OMe}, \mathrm{OAc}, \mathrm{NHCOMe}, \mathrm{Csp} p^{2}$

Scheme 8. Regioselective difunctionalization of alkenes.

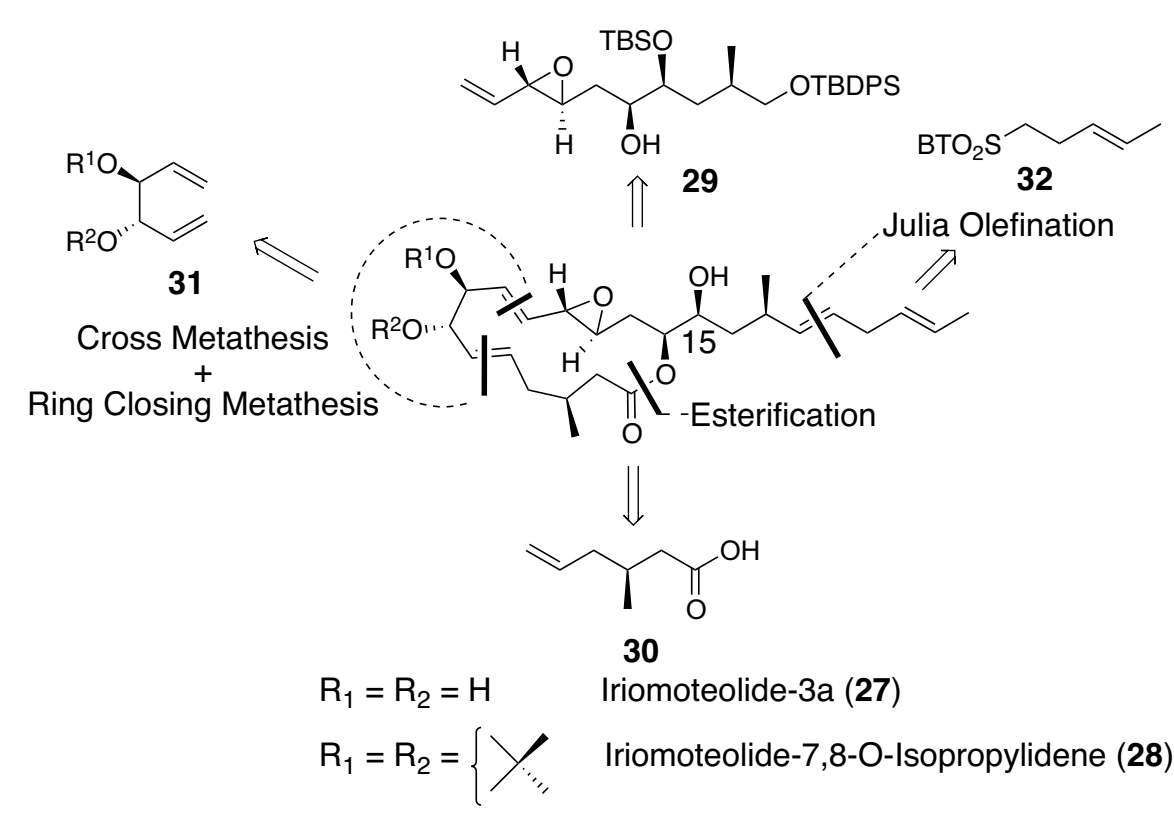

Scheme 9. Retrosynthesis of Iriomoteolide-3a (27). gold-catalyzed alkene functionalizations is much lower than those of alkynes. We wondered whether or not $\mathrm{Au}(\mathrm{I}) / \mathrm{Au}(\mathrm{III})$ redox catalytic cycles could be employed towards the efficient difunctionalization of non-activated olefins. Upon coordination of the double bond to gold, an intramolecular nucleophilic attack could occur in the presence of a suitable electron-donor atom generating an alkyl-gold intermediate, which could then be functionalized further. The reaction of 4-penten-1-amines (26) with a catalytic amount of gold(I) and a stoichiometric amount of oxidant in the presence of external nucleophiles (water, alcohols or acetonitrile) delivered the corresponding amino-alcohols, ethers, esters or amides in excellent yields (Scheme 8). [20] These transformations were highly regioselective favoring the formation of 6-endo products. Two possible mechanistic scenarios arise for these transformations: either the incorporation of the external nucleophile occurs by a reductive elimination on a Csp ${ }^{3}$-gold(III) intermediate or, alternatively, two consecutive nucleophilic substitution reactions through a highly reactive aziridinium intermediate, could also explain the formation of the observed products. Preliminary mechanistic studies seem to support the latter reaction pathway.

\section{Natural Products as Chemical Probes}

Although more than $50 \%$ of marketed drugs are based on natural products (NPs) or derivatives thereof, [21] the small quantities in which these valuable molecules can be obtained from their natural sources poses a big limitation to future NP-based drug development. Laboratory synthesis is key, not only to provide enough quantities of NPs for biological applications, but also to optimize these compounds establishing the adequate pharmacological profiles for future use as clinical tools. ${ }^{[22]}$

Marine ecosystems are a very prolific source of secondary metabolites. Recently, the Amphidinium strain HYA024 was found to produce structurally unique cytotoxic compounds such as Iriomoteolides 1a-c, as well as a rare 15-membered macrolide, Iriomoteolide-3a (27). [23] Compound 27 and its acetonide derivative $\mathbf{2 8}$ were profiled against a small panel of cancer cell 
Table 1. Growth inhibitory activity of Irio-derivatives

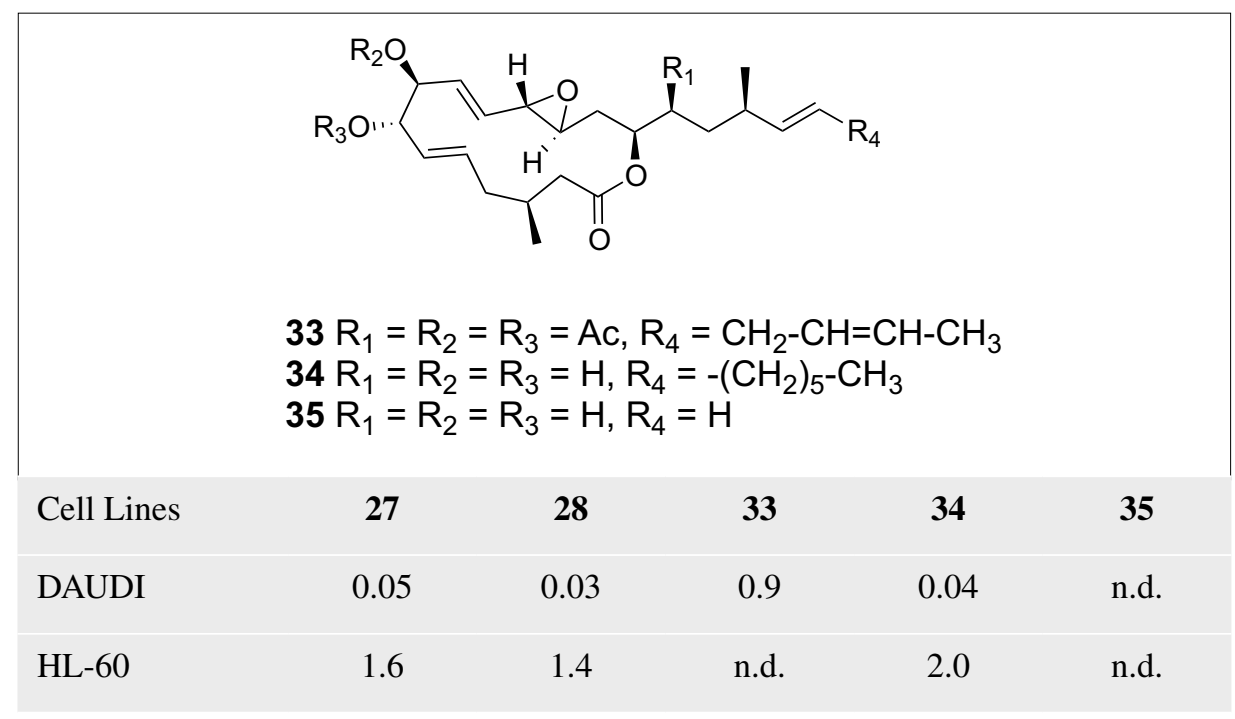

$\mathrm{GI}_{50}$ values in range $\mu \mathrm{g} / \mathrm{mL}$; n.d.= not determined; no activity was observed even at $10 \mu \mathrm{M}$.

lines, with promising results. We decided to embark on a total synthesis campaign to first confirm the assigned structure of these novel macrolides and second to exhaustively investigate their biological activity. From a synthetic perspective, these molecules offered interesting challenges, as eight chiral centers, four of them located in allylic positions, needed to be efficiently installed.

\subsection{Total Synthesis of Iriomoteolide-3a}

Our retrosynthesis of $\mathbf{2 7}$ is shown in Scheme 9. The $C_{2}$ symmetry of the 1,2-diol moiety inspired a strategy in which the 1,5-diene would be introduced through a Cross-Metathesis/Ring-Closing Metathesis sequence. In only one step the 15 -membered ring macrolide would be assembled in a stereoselective manner using fragment 31, easily derived from L-tartaric acid. An intermolecular esterification reaction was proposed to connect fragments 29 and 30. Fragment 32 would be incorporated last by means of a Julia-Kocienski olefination reaction to avoid the undesired conjugation of the two double bonds. In addition, we also aimed to synthesize analogues of the molecule by late-stage coupling of different side chains. The four building blocks 29-32 were synthesized and assembled according to this strategy. ${ }^{[22]}$ Our next goal was to perform a small structure-activity relationship study (SAR) to understand the influence of the different structural motifs on the biological activity of these molecules. Thus, the 1,2-diol in 27 was protected as an acetal $(\mathbf{2 8})$ and all the hydroxy groups in $\mathbf{2 7}$ were acetylated leading to peracetylated derivative $\mathbf{3 3}$. The side chain was used to diversify the macrolide blueprint: longer, and thus more lipophilic (34) as well as shorter side chains (35) were incorporated via Julia and Wittig olefination reactions, respectively.

The cell growth inhibitory activity of the compounds 27, 28, 33, 34 and 35 was evaluated in two different types of cancer cells: DAUDI (lymphoma) and HL-60 (leukemia) (Table 1).

Compounds 27 and 28 showed a high activity against lymphoma cells $\left(\mathrm{GI}_{50}=\right.$ 0.05 and $0.03 \mu \mathrm{g} / \mathrm{mL}$, respectively) confirming the preliminary results published in the original work reporting the isolation of these molecules. ${ }^{[23]}$ We assume that the hydroxy group in $\mathrm{C}(15)$ plays a central role in the bioactivity of these compounds as a dramatic drop in the activity of $\mathbf{3 3}$ was observed. The truncation of the side chain causes the total loss of the anti-proliferative activity in compound $\mathbf{3 5}$, showcasing the importance of the side chain in the biological activity of these macrolides. [24]

In summary, the first total synthesis of Iriomoteolide-3a (27) and the confirmation of its proposed structure was reported using a stereoselective sequence of CrossMetathesis/Ring Closing Metathesis as key synthetic disconnection. A small collection of synthetic irio-analogues was prepared and their anti-proliferative activity evaluated revealing which are the suitable points for chemical edition in the original molecule.

\section{Conclusions}

The combination of new methodologies with classical tools of organic chemistry offers an excellent platform for the design and synthesis of complex molecules. Our research group will continue applying a multidisciplinary approach to design, synthesize and utilize organic molecules as chemical probes in the study of relevant biological processes.

\section{Acknowledgements}

I would like to thank the Swiss Chemical Society for the award of the Werner Prize 2013, which I am honored to share with my esteemed colleague Prof. Clément Mazet. The little or big accomplishments that this award generously recognized stem less from this principal investigator but most from the enthusiastic contribution of hard-working individuals (Master, $\mathrm{PhD}$ students and Post-docs) that decided to join our group and devote their energies to a common passion, chemistry. To each single one of them (- past: Olivia, Teresa, Karine, David, Riccardo, Noam, Xiaogen, Karolin, Pascal, Tobias, - and present: Manuel, Andrea, Martin, Manuela, Luis, Roopender, Giulia, Wangqing, Noelia, Patricia, Philipp, Maria, Ana, Estibaliz and Fabian) I will always be grateful. Financial support from the University of Zürich, the Swiss National Science Foundation, Givaudan AG, Novartis and the European Union through an ERC Starting Grant is kindly acknowledged.

Received: August 6, 2013

[1] a) K. Lafleur, D. Z. Huang, T. Zhou, A. Caflisch, C. Nevado, J. Med. Chem. 2009, 52, 6433; b) D. Huang, T. Zhou, K. Lafleur, C. Nevado, A. Caflisch, Bioinformatics 2010, 26, 198; c) H. Zhao, J. Dong, K. Lafleur, C. Nevado, A. Caflisch, J. Med. Chem. Lett. 2012, 3, 834; d) K. Lafleur, D. Huang, T. Zhou, A. Caflisch, C. Nevado, J. Med. Chem. 2013, 56, 84.

[2] For a previous account on our program, see: D. Garayalde, T. de Haro, K. Lafleur, C. Nevado, An. Quím. 2011, 107, 297.

[3] a) P. Pyykkö, Angew. Chem. Int. Ed. 2004, 43, 4412; b) P. Pyykkö, Ann. Rev. Phys. Chem. 2012, 63, 45; c) D. J. Gorin, F. D. Toste, Nature 2007, 446, 395; d) A. Leyva-Perez, A. Corma Angew. Chem. Int. Ed. 2012, 51, 614.

[4] a) A Fürstner, P. W. Davies, Angew. Chem. Int. Ed. 2007, 46, 3410; b) E. Jiménez-Nuñez, A. M. Echavarren, Chem. Rev. 2008, 108, 3326; c) A. K. S. Hashmi, Angew. Chem. Int. Ed. 2010, 49 , 5232.

[5] a) N. Marion, S. P. Nolan, Angew. Chem. Int. Ed. 2007, 46, 2750; b) C. Nevado, Chimia 2010, $64,247$.

[6] a) X. Huang, T. de Haro, C. Nevado, Chem. Eur. J. 2009, 15, 5904; b) T. de Haro, E. GómezBengoa, R. Cribiú, X. Huang, C. Nevado, Chem. Eur. J. 2012, 18, 6811.

[7] a) Y. Zou, D. Garayalde, Q. Wang, C. Nevado, A. Goeke, Angew. Chem. Int. Ed. 2008, 47, 10110 ; b) D. Garayalde, E. Gómez-Bengoa, X. Huang, A. Goeke, C. Nevado, J. Am. Chem. Soc. 2010, 132, 4720.

[8] D. Garayalde, C. Nevado, ACS Catal. 2012, 2, 1462.

[9] M. J. Johansson, D. J. Gorin, S. T. Staben, F. D. Toste, J. Am. Chem. Soc. 2005, 127, 18002.

[10] D. Garayalde, K. Krüger, C. Nevado, Angew. Chem. Int. Ed. 2011, 50, 911.

[11] S. G. Bratsch, J. Phys. Chem. Ref. Data 1989, $18,1$.

[12] a) C. González-Arellano, A. Corma, M Iglesias, F. Sánchez, J. Catal. 2006, 238, 497; 
b) A. Corma, E. Gutiérrez-Puebla, M. Iglesias, A. Monge, S. Pérez-Ferreras, F. Sánchez, Adv. Synth. Catal. 2006, 348, 1899; c) C. GonzálezArellano, A. Abad, A. Corma, H. García, M. Iglesias, F. Sánchez, Angew. Chem. Int. Ed. 2007, 46, 1536; d) P. Li, L. Wang, M. Wang, F. You, Eur. J. Org. Chem. 2008, 5946; e) C. González-Arellano, A. Corma, M. Iglesias, F. Sánchez, Eur. J. Inorg. Chem. 2008, 1107.

[13] a) T. Lauterbach, M. Livendahl, A. Rosell, P. Espinet, A. M. Echavarren, Org. Lett. 2010, 12, 3006; b) A. Corma, R. Juárez, M. Boronat, F. Sánchez, M. Iglesias, H, García, Chem. Commun. 2011, 47, 1446.

[14] a) H. A. Wegner, Chimia 2009, 63, 44; b) P. García, M. Malacria, C. Aubert, V. Gandon, L. Fensterbank, ChemCatChem 2010, 2, 493; c) M. N. Hopkinson, A. D. Gee, V. Gouverneur, Isr. J. Chem. 2010, 50, 675; d) M. N. Hopkinson, A D. Gee, V. Gouverneur, Chem. Eur. J. 2011, 17, 8248; T. de Haro, C. Nevado, Synthesis 2011, 16, 2530; f) H. A. Wegner, Angew. Chem. Int. Ed. 2011, 50, 8236.

[15] T. de Haro, C. Nevado, J. Am. Chem. Soc. 2010 , $132,1512$.

[16] P. Kirsch in 'Modern Fluoroorganic Chemistry: Synthesis Reactivity Applications', Ed. P. Kirsch, Wiley-VCH, Weinheim, 2004.

[17] K. M. Engle, T.-S. Mei, X. Wang, J.-Q. Yu, Angew. Chem. Int. Ed. 2011, 50, 1478.

[18] T. de Haro, C. Nevado, Chem. Commun. 2011, 47, 248.

[19] T. de Haro, C. Nevado, Adv. Synth. Catal. 2010, 352, 2767.

[20] T. de Haro, C. Nevado, Angew. Chem. Int. Ed. 2011, 50, 906 .

[21] a) K. C. Nicolau, E. J. Sorensen in 'Classics in Total Synthesis', Weinheim, Wiley-VCH, 1996; b) K. C. Nicolau, S. A. Snyder, 'Classics in Total Synthesis II', Weinheim, Wiley-VCH, 2003.

[22] W. R. Strohl, Drug Discovery Today 2000, 5, 39.

[23] a) J. Kobayashi, M. Tsuda, Nat. Prod. Rep. 2004, 21, 77; b) J. Kobayashi, T. Kubota, J. Nat. Prod. 2007, 70, 451.

[24] a) R. Cribiú, C. Jäger, C. Nevado, Angew. Chem. Int. Ed. 2009, 48, 8780; b) For the synthesis of skeleton of Iriomoteolide-3a, see: C. R. Reddy, G. Dharmapuri, N. N. Rao, Org. Lett. 2009, 11, 5730 . 